\title{
Chronic insomnia cases detection with a help of Athens Insomnia Scale and SF-36 health survey
}

\author{
P. Wąsiewicz ${ }^{a}$, M. Skalski ${ }^{b}$, M. Fornal-Pawlowska $^{b}$ \\ ${ }^{a}$ Institute of Electronic Systems, Warsaw University of Technology \\ ${ }^{b}$ Outpatient Sleep Disorders Clinic, Department of Psychiatry, Medical University of Warsaw, \\ WUM
}

\begin{abstract}
Standardization of the diagnostic process of insomnia is a highly important task in clinical practice, epidemiological considerations and treatment outcomes assessment. In this paper we describe standard surveys relationships within cluster groups with the same insomnia degrees.
\end{abstract}

Keywords: insomnia, quality life profiles, clustering, random forest

\section{INTRODUCTION}

Sleep disorders are growing problems with serious consequences. 30-40\% of adults have experienced some level of insomnia - sleep disorders of initiating and maintaining sleep. 10-15\% of adults report chronic and severe insomnia. More and more people are ill and complain. Insomnia is connected with the complaint of falling asleep difficulty, staying asleep difficulty, poor quality sleep and is associated with distress and impaired functioning. There are many causes of insomnia including medical diseases, psychiatric disorders, behavioral factors, circadian dysrhythmias, primary sleep disorders. ${ }^{1}$ Daytime complaints about insomnia are fatigue, sluggishness, sleepiness, somatic complaints (aches \& pains), stress about poor sleep, mood disturbances, poor concentration, impaired performance.

The Athens Insomnia Scale (AIS) is the instrument designed for quantitative measurement of the severity of insomnia based on the ICD-10 (International Statistical Classification of Diseases and Related Health Problems) criteria and is intended to record own patients assessment of any sleep difficulty they might have experienced. ${ }^{2,3}$ The reliability and validity of the polish version were confirmed. ${ }^{4}$

The 36-item Short-Form Health Survey (SF-36) is a self-report measure of life quality related to health. ${ }^{5,6}$ It comprises 36 questions that assess eight health dimensions: physical functioning, role limitations due to physical problems, bodily pain, general health, vitality (energy), social functioning, role limitation due to emotional problems and mental health (emotional wellbeing). Scores on each dimension can range from 0 to 100 . Higher score indicated a better quality of life. The SF-36 was adapted in more than 50 countries as part of the International Quality of Life Assessment (IQOLA) Project. The reliability and validity of the polish version were confirmed. ${ }^{7}$

In this paper we check dependency of the Polish version of the Athens Insomnia Scale (AIS) on the Polish version of the quality of life survey (SF-36).

\section{DATA RETRIEVAL METHODOLOGY}

A decision tree is a tree in which each branch node represents a choice between a number of alternatives, and each leaf node represents a classification or decision. A decision tree takes as input an object or situation described by a set of properties, and outputs a yes/no decision. Functions with a larger range of outputs can also be represented. $^{8}$ The so called random forest is a modified classification tree bagging with randomly chosen number of attributes for each node test. 


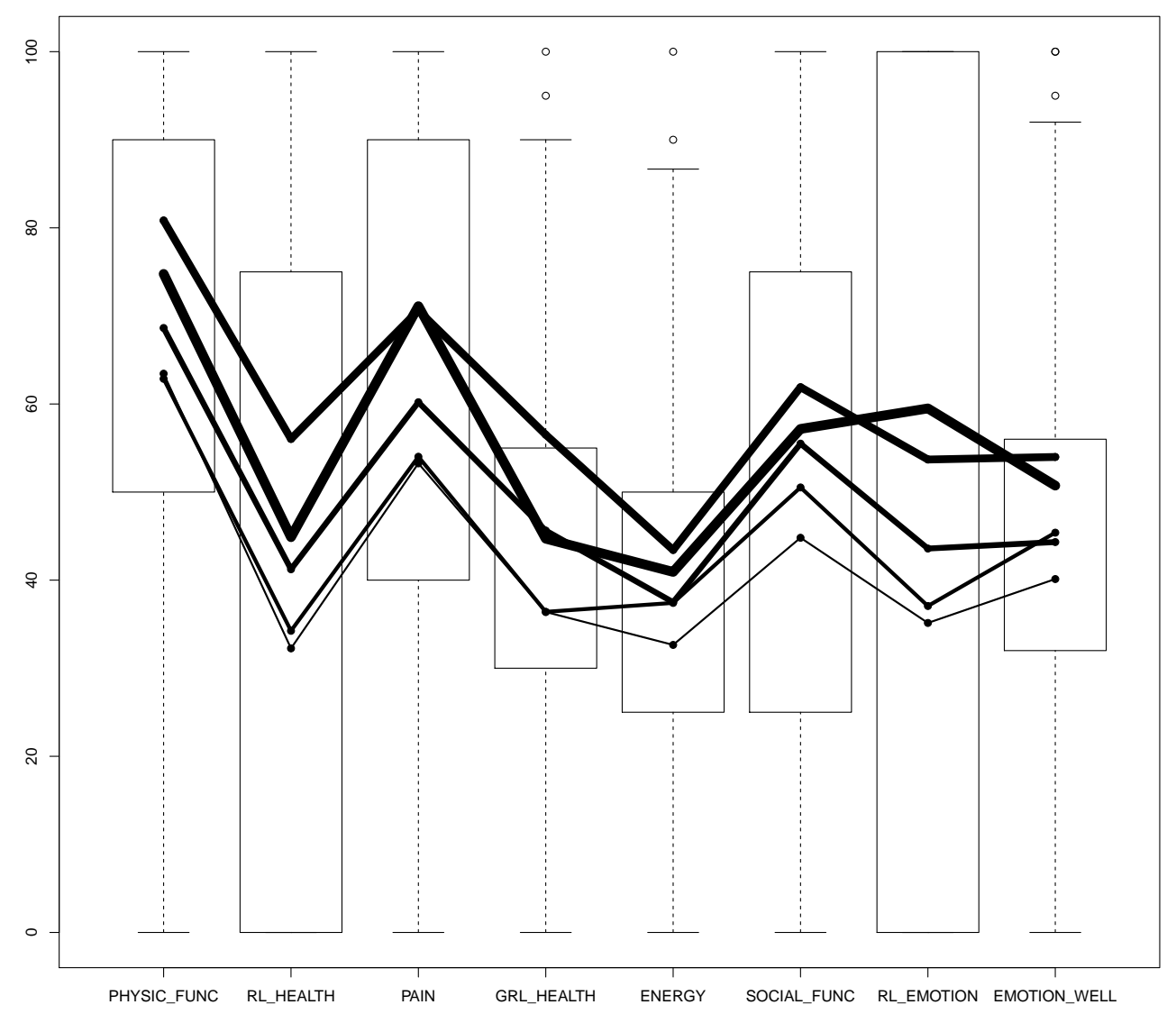

Figure 1. The five patient groups with different profiles

Data clustering is the unsupervised clustering of patterns e.g. observations, data items, feature vectors into groups, so the data in each subset share common trait like proximity according to some defined distance measure. ${ }^{9}$ In general, a grouping is method to show similar objects near in features space. Data clustering algorithms can be hierarchical or partitional. Hierarchical algorithms find successive clusters using previously established clusters, whereas partitional algorithms determine all clusters at once. Hierarchical algorithms can be agglomerative ("bottom-up") or divisive ("top-down"). Agglomerative algorithms begin with each element as a separate cluster and merge them into successively larger clusters. Divisive algorithms begin with the whole set and proceed to divide it into successively smaller clusters.

A key step in a hierarchical clustering is to select a distance measure. A simple measure is Manhattan measure equal to the sum of absolute differences for each variable. A more common measure is Euclidean distance, computed by finding the square of the distance between each variable, summing the squares, and finding the square root of that sum. A property of the Euclidean space is that distances are symmetric (the distance from object A to B is the same as the distance from B to A). Given a distance measure, elements can be combined. Hierarchical clustering builds (agglomerative nesting hierarchical clustering), or breaks up (divisive analysis clustering), a hierarchy of clusters.

Further author information: (Send correspondence to Michal Skalski)

M. Skalski, e-mail: michal.skalski@wum.edu.pl

M. Fornal-Pawlowska, e-mail: m.fornal@wp.pl

P. Wasiewicz, e-mail: pwasiewi@elka.pw.edu.pl 


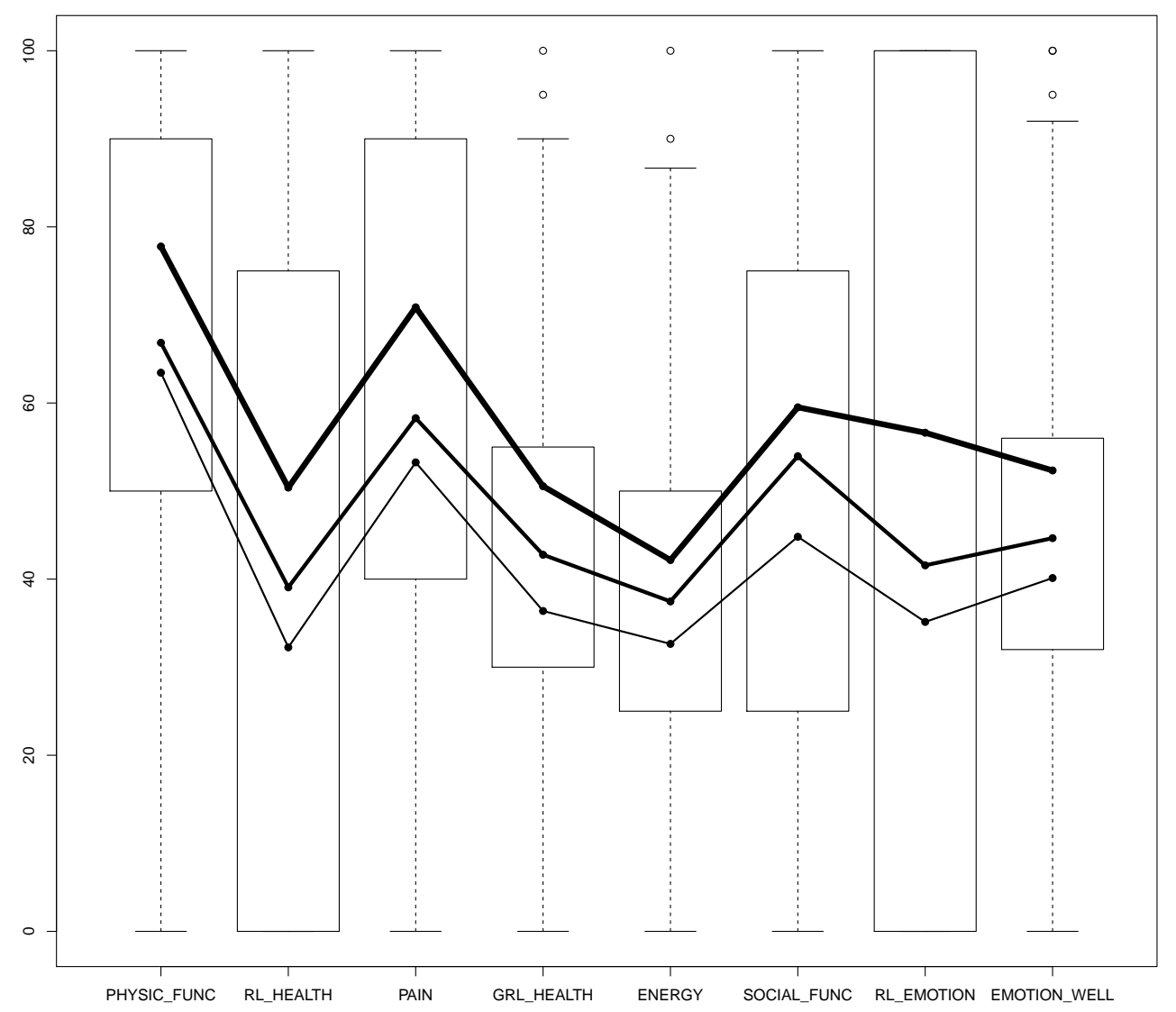

Figure 2. The three main groups of level II, I and new insomnia

The traditional representation of this hierarchy is a tree data structure (called a dendogram), with individual elements at one end and a single cluster with every element at the other. Agglomerative algorithms begin at the top of the tree, whereas divisive algorithms begin at the bottom. Cutting the tree at a given height will give a clustering at a selected precision.

In partitional clustering the k-means algorithm assigns each point to the cluster whose center (also called centroid) is nearest. The center is the average of all the points in the cluster - that is, its coordinates are the arithmetic mean for each dimension separately over all the points in the cluster.

In fuzzy clustering (fuzzy analysis clustering), each point has a degree of belonging to clusters, as in fuzzy logic, rather than belonging completely to just one cluster. Thus, points on the edge of a cluster, may be in the cluster to a lesser degree than points in the center of cluster. For each point its coefficients of being in the clusters are computed. As in k-means algorithm the minimum is a local minimum and the results depend on the initial choice of weights.

The solution space can be visualized in two dimensions after scaling based e.g. on principle component analysis (PCA), which transform real dimensions to artificial ones, where first ones have the most correlations within and the rest of dimensions may be omitted with small resulting errors.

The multidimensional space can be also visualized in two "artificial" dimensions after scaling based e.g. on the general dissimilarity coefficient of Gower ${ }^{10}$ and Kruskal nonmetric MDS, ${ }^{11}$ where each point dissimilarities with other ones are transferred into two dimensional spacial distances between them. 


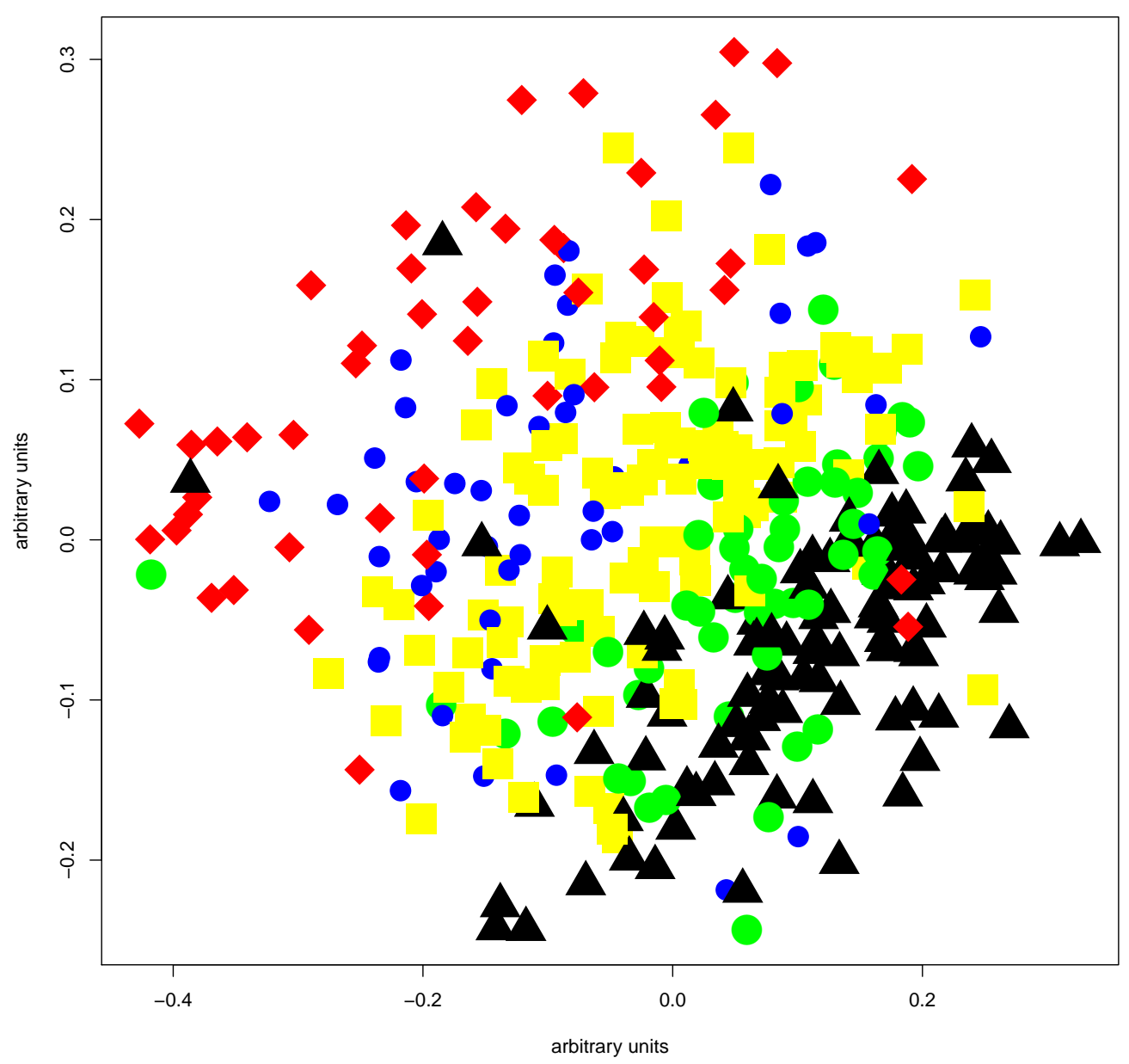

Figure 3. Multidimensional (all SF-36 and AIS parameters) scaling plot of patients: black triangles - patients from the cluster no 1 with severe insomnia are dominating on the right, big cirles - the cluster no 2 , squares - 3 , rhombs - 4 , small cirles - 5

\section{DATA RETRIEVAL RESULTS}

Data sets were obtained from 377 patients of Outpatient Sleep Disorders Clinic at WUM at the beggining of their therapy. It is obligatory for every patient to fill all necessary surveys at the treatment beginning, but only 377 patients full data was typed into computer database to test new ideas e.g. insomnia is considered to be the only one typical disease for all people with all sleep disorders, maybe there is an another insomnia kind. In this paper we try to find different SF-36 profiles for peoples with three main problems: falling asleep difficulties, awakenings throughout the night, waking up too early in the morning connected with three AIS questions with four values: 0 - no problem,1,2,3.

\subsection{Quality life parameter importance}

Importance of SF-36 scales in recognizing the mentioned three main insomnia problems was detected with a help of $\mathrm{R}$ statistical environment ${ }^{12}$ and randomForest (a randomForest function), party (a cforest function) packages.

The randomForest function creates very good classifiers, even for small $\mathrm{n}$ large $\mathrm{p}$, where $\mathrm{n}$ - number of rows, p - columns. The randomForest (for minimum 30 trees) SF-36 parameters importance was the same for all three insomnia problems (contrary to physician experience): the highest importance has general health scale, the 
second - emotional wellbeing scale, and the third - energy scale connected with depression. Falling asleep and waking-ups were also connected with general health and emotional wellbeing.

The cforest function is a random forest extended with conditional inference. Conditional variable importance "breaks" the variable's bond to the response by randomly shuffling the values of a given independent variable. Then, the difference of the model accuracy before and after the random permutations, averaged over all trees in the forest, tells us how important that predictor is for determining the outcome. The cforest (for 100 trees) had different importance lists for some (but not for every one) problems, but its classification error was higher.

After tweaking cforest parameters (making cforest more similar in action to typical random forest) and using conditional importance for different population numbers (50, 100, 250, 277, 1000 decision trees), the cforest classificator error was much more smaller and all main insomnia problems has different importance lists: for falling asleep - physical functioning, and role limitation due to emotional problems; for night awakenings - role limitations due to physical problems, social functioning; for too early morning waking-ups - general health, energy.

\subsection{Clustering}

For clustering several procedures from R environment were used: diana (DIvisive ANAlysis Clustering), fanny (Fuzzy Analysis Clustering with squared dissimilarity input matrix), hclust (Hierarchical Clustering with squared dissimilarity input matrix), agnes (Agglomerative Nesting - Hierarchical Clustering with dissimilarity input matrix), kmeans (K-Means Clustering), cutree (dendogram cutting into groups function used for diana, hclust, agnes). A cluster number was set to two, three or four clusters. Only three columns of the given data with three AIS attributes (difficulties falling asleep, staying asleep during the nigth and early in the morning) were used by the mentioned above procedures. Their values range from 0 to 3 .

During computation it appeared that the patients with advanced insomnia dominated the whole population of 377 individuals (black dots and ligth gray squares in Fig. 3) and the mentioned algorithms could not find the correctly separated clusters (with separated, not crossed, quality life profile lines). First we removed all people with the same values 2 and 3 of difficulties falling asleep, staying asleep during the night and early in the morning, but it turned out that it is enough to remove 35 people with the same values of 2 (all medium difficulties). On the rest of 342 people four clusters were calculated. The best solution of finding different SF-36 profile groups with different degrees of falling and staying asleep problems was computed by the fanny function for four clusters. The fifth cluster was artificially created from people with the same very high values 3 of all sleep disorders and was called the cluster no 1 and the rest of cluster numbers encreased by one. The removed earlier 35 patients were added to the most similiar to them cluster no 3 . The values of difficulty levels in all five groups are put in Table 1.

In every cluster means of parameters were computed and for all clusters their statistical significance (panova - p value) was determined with a use of anova tests for each parameter. Finally, standard deviation (sd) for every attribute was calculated as is provided in Table 2. The column called sd/dmean contains means of standard deviations divided by substracted neighbouring cluster means. The table is ordered by the mentioned column values and quality of life parameters in the first rows are better divided into separated groups.

Cluster SF-36 profiles (means) were depicted in Fig. 1. Boxes in Figs. 1 and 2 are made of the first and third quartiles. The first quartile called lower quartile cuts off lowest $25 \%$ of data. The second quartile called median divides the ordered data set into two halves. The third quartile called upper quartile cuts off highest $25 \%$ of data, or lowest $75 \%=75$ th percentile. The first group (the thinnest line) consists of people with all high level values 3 of all three difficulties of falling and staying asleep during night and too early in the morning. The second group consists of people with more than medium difficulties of falling asleep and all awakening problems. The third group is for people with more than medium difficulties of falling asleep and medium ones of staying asleep during night and too early in the morning. The fourth group has only medium problems with night awakenings. The last fifth cluster (the thickest line) has only medium problems with all awakening sorts. Thus, the fourth and fifth cluster has no problem with falling asleep.

The group no 1 with all severe sleep disorders is a severe insomnia level II. ${ }^{13}$ The groups no 2 and 3 with medium falling asleep difficulties form together a medium insomnia level I. ${ }^{13} \mathrm{~A}$ light level new kind of insomnia 
Table 1. The value table of five clusters

\begin{tabular}{rrrrr}
\hline & 0 & 1 & 2 & 3 \\
\hline falling asleep in the cluster no 1 & 0 & 0 & 0 & 107 \\
night awakening in the cluster no 1 & 0 & 0 & 0 & 107 \\
morning awakening in the cluster no 1 & 0 & 0 & 0 & 107 \\
falling asleep in the cluster no 2 & 0 & 0 & 27 & 26 \\
night awakening in the cluster no 2 & 0 & 0 & 16 & 37 \\
morning awakening in the cluster no 2 & 0 & 0 & 29 & 24 \\
falling asleep in the cluster no 3 & 0 & 0 & 60 & 58 \\
night awakening in the cluster no 3 & 4 & 21 & 84 & 9 \\
morning awakening in the cluster no 3 & 18 & 26 & 69 & 5 \\
falling asleep in the cluster no 4 & 38 & 5 & 6 & 0 \\
night awakening in the cluster no 4 & 10 & 23 & 15 & 1 \\
morning awakening in the cluster no 4 & 32 & 17 & 0 & 0 \\
falling asleep in the cluster no 5 & 20 & 30 & 0 & 0 \\
night awakening in the cluster no 5 & 0 & 6 & 24 & 20 \\
morning awakening in the cluster no 5 & 1 & 11 & 26 & 12 \\
\hline
\end{tabular}

Table 2. ANOVA Table of five clusters

\begin{tabular}{|c|c|c|c|c|c|c|c|c|c|}
\hline & & 1 & 2 & 3 & 4 & 5 & sd/dmean & p_anova & $\mathrm{sd}$ \\
\hline 8 & SOCIAL_FUNC & 44.81 & 50.52 & 55.50 & 61.91 & 57.17 & 3.70 & 0.0013126 & 27.17 \\
\hline 1 & EMOTION_WELL & 40.12 & 45.39 & 44.32 & 53.98 & 50.73 & 3.99 & 0.0001587 & 19.23 \\
\hline 7 & RL_HEALTH & 32.25 & 34.25 & 41.23 & 56.03 & 44.88 & 4.41 & 0.0068554 & 39.77 \\
\hline 6 & RL_EMOTION & 35.14 & 37.06 & 43.59 & 53.70 & 59.50 & 4.86 & 0.0024672 & 41.20 \\
\hline 5 & PHYSIC_FUNC & 63.44 & 62.86 & 68.63 & 80.84 & 74.77 & 5.40 & 0.0001904 & 25.27 \\
\hline 2 & ENERGY & 32.65 & 37.42 & 37.49 & 43.42 & 40.93 & 17.31 & 0.0033890 & 17.61 \\
\hline 4 & PAIN & 53.26 & 54.01 & 60.20 & 70.62 & 71.11 & 18.80 & 0.0001776 & 29.11 \\
\hline 3 & GRL_HEALTH & 36.38 & 36.40 & 45.63 & 56.51 & 44.70 & 76.42 & 0.0000001 & 21.17 \\
\hline
\end{tabular}

is made of two next groups no 4 and 5 only with awakenings problems. Three different life quality profiles are shown in Fig. 2: the new insomnia kind as a thick line, the level II one as the thinnest line. Anova $p$ values of these three groups are in Table 3. As you can see, all quality life parameters are very good separated with very low $\mathrm{p}$ anova values and almost the same low $\mathrm{sd} / \mathrm{dmean}$ values.

\section{SUMMARY}

We found different important predictors for each main insomnia problem. We found 5 groups with different, separated profiles and joint them together to form not only the level I and II insomnias, but also a new kind of light insomnia with only awakenings problems. We proved that insomnia is associated with reduced mental health, vitality, and social functioning, but at different degrees for each insomnia level.

Table 3. ANOVA Table of three insomnia kinds

\begin{tabular}{llrrrrrr}
\multicolumn{7}{c}{ Table 3. ANOVA Table of three insomnia kinds } \\
\hline & & 1 & 2 & 3 & sd/dmean & p_anova & sd \\
\hline 3 & GRL_HEALTH & 36.38 & 42.77 & 50.55 & 2.26 & 0.0000073 & 21.17 \\
1 & EMOTION_WELL & 40.12 & 44.65 & 52.34 & 2.47 & 0.0000194 & 19.23 \\
2 & ENERGY & 32.65 & 37.47 & 42.17 & 2.78 & 0.0004835 & 17.61 \\
4 & PAIN & 53.26 & 58.28 & 70.87 & 2.85 & 0.0000341 & 29.11 \\
8 & SOCIAL_FUNC & 44.81 & 53.96 & 59.51 & 2.89 & 0.0003610 & 27.17 \\
6 & RL_EMOTION & 35.14 & 41.57 & 56.62 & 3.25 & 0.0005426 & 41.20 \\
5 & PHYSIC_FUNC & 63.44 & 66.84 & 77.77 & 3.32 & 0.0000882 & 25.27 \\
7 & RL_HEALTH & 32.25 & 39.07 & 50.39 & 3.43 & 0.0040522 & 39.77 \\
\hline
\end{tabular}


In our future research it will be a chance for finding some extraordinary and remarkable relashionships between patients profile and further pharmacological treatment, which helps in chronic insomnia diagnosis.

\section{REFERENCES}

[1] CostaeSilva, J. A., Chase, M., Sartorius, N., and Roth, T., "Special report from Symposium held by the World Health Organization and the World Federation of Sleep Research Societies: An overview of insomnias and related disorders - recognition, epidemiology, and rational management.," Sleep 19, 412-416 (1996).

[2] Soldatos, C. R., Dikeos, D. G., and Paparrigopoulos, T. J., "Athens Insomnia Scale: validation of an instrument based on ICD-10 criteria.," Journal of Psychosomatic Research 48, 555-560 (2000).

[3] Soldatos, C. R., Dikeos, D. G., and Paparrigopoulos, T. J., "The diagnostic validity of the Athens Insomnia Scale.," Journal of Psychosomatic Research 55, 263-267 (2003).

[4] Szelenberger, W., Wolynczyk-Gmaj, D., and Fornal-Pawlowska, M., "Validation of the Polish version of the Athens Insomnia Scale.," Psychiatria Polska 45(2), 211-219 (2011).

[5] McHorney, C. A., Ware, J. E., Lu, J. F., and Sherbourne, C. D., "The MOS 36-item short-form health survey (SF-36): III. tests of data quality, scaling assumptions, and reliability across diverse patient groups.," Med. Care 32, 40-66 (1994).

[6] Ware, J. E. and Gandek, B., "Overview of the SF-36 health survey and the international quality of life assessment (IQOLA) project.," J. Clin. Epidemiol. 51, 903-912 (1998).

[7] Marcinowicz, L. and Sienkiewicz, J., "Badanie trafnosci i rzetelnosci polskiej wersji kwestionariusza SF-36: wyniki wstepne.," Przeglad Lekarski 60, 103-106 (2003).

[8] Russell, S. and Norvig, P., [Artificial Intelligence: A Modern Approach.], Prentice Hall (2009).

[9] Kaufman, L. and Rousseeuw, P. J., [Finding Groups in Data: An Introduction to Cluster Analysis.], J. Wiley and Sons, Inc., New York (1990).

[10] Gower, J. C., "A general coefficient of similarity and some of its properties.," Biometrics 27, 623-637 (1971).

[11] Kruskal, J. B., "Multidimensional scaling by optimizing goodness of fit to a nonmetric hypothesis.," Psychometrika 29, 1-27 (1964).

[12] Venables, W. N. and Ripley, B. D., [Modern applied statistics with S], Springer-Verlag, New York, 4th ed. (2002).

[13] Terzano, M. G., Parrino, L., Cirignotta, F., L. F.-S., Gigli, G., Rudelli, G., and Sommacal, S., "Studio Morfeo: insomnia in primary care, a survey conducted on the Italian population.," Sleep 5, 67-75 (2004). 